\title{
Etude Chimique et Micro-Cartographique De Roches Carbonatées : Cas Des Calcaires Lumachelliques De l'Oligocène Inferieur Du Bassin Emergé de Côte d'Ivoire
}

\author{
Assalé Fori Yao Paul, PhD \\ Université Félix Houphouët Boigny, UFR des Sciences de la Terre et des \\ Ressources Minières, Abidjan, Côte d'Ivoire \\ Gbangbot Jean Michel Kouadio, PhD \\ Université Lorougnon Guédé de Daloa, Côte d'Ivoire \\ Kessé Touvalé Marcel, PhD \\ Institut National Polytechnique Houphouët Boigny, \\ Yamoussoukro, Côte d'Ivoire \\ Kplohi Yaba Hervé, Msc \\ Féa Isaac, Msc \\ PETROCI, Centre d'Analyses et de Recherche (CAR), \\ Abidjan, Côte d'Ivoire
}

Doi:10.19044/esj.2021.v17n14p52

Submitted: 13 October 2020

Accepted: 23 February 2021

Published: 30 April 2021
Copyright 2021 Author(s)

Under Creative Commons BY-NC-ND

4.0 OPEN ACCESS

Cite As:

Yao Paul A.F., Michel Kouadio G.J., Marcel T.K., Yaba Hervé K. \& Isaac F. (2021). Etude Chimique et Micro-Cartographique De Roches Carbonatées : Cas Des Calcaires Lumachelliques De l'Oligocène Inferieur Du Bassin Emergé de Côte d'Ivoire. European Scientific Journal, ESJ, 17(14), 52. https://doi.org/10.19044/esj.2021.v17n14p52

\section{Resume}

Dans le présent travail, une étude minéralogique et cartographique chimique des calcaires lumachelliques de l'Oligocène inférieur du bassin émergé de Côte d'Ivoire a été effectuée. Les analyses ont été réalisées au M.E.B, équipé d'un spectromètre à diffusion d'énergie. L'objectif est de comprendre les processus post-diagénétiques qui se sont produits dans les calcaires lumachelliques au cours de la transgression de l'Oligocène inférieur. Les résultats ont montré que les calcaires lumachelliques sont composés de plus de $80 \%$ de calcium qui sont accompagnés par ordre d'abondance de silicium $(6,73 \%)$, de soufre $(1,94 \%)$, de fer $(1,62 \%)$, d'aluminium $(1,57 \%)$ et de magnésium $(1,44 \%)$. Les résultats révèlent également qu'au cours de la 
transgression de l'Oligocène inférieur, des inclusions minéralogiques ont été formées dans l'ordre chronologique suivant : la glauconite et la pyrite. Durant cette transgression une silicification (quartzification) de certains bioclastes s'est produite et cela entre les profondeurs $3000 \mathrm{~m}$ et la zone de compensation des carbonates (CDD) expliquant ainsi la présence de silice dans ces calcaires par la micro-cartographie chimique. Ce nouvel environnement de dépôt (marin profond) a occasionné encroûtement de leur surface donnant ainsi un hardground.

Mots-clés : Silicification, Calcaire lumachellique, Micro-cartographie chimique

\title{
Chemical and Micro-Mapping Study of Carbonate Rocks: Case of Shelly Limestone of Lower Oligocene of Côte d'Ivoire Onshore Sedimentary Basin
}

\author{
Assalé Fori Yao Paul, PhD \\ Université Félix Houphouët Boigny, UFR des Sciences de la Terre et des \\ Ressources Minières, Abidjan, Côte d'Ivoire \\ Gbangbot Jean Michel Kouadio, PhD \\ Université Lorougnon Guédé de Daloa, Côte d'Ivoire \\ Kessé Touvalé Marcel, PhD \\ Institut National Polytechnique Houphouët Boigny, \\ Yamoussoukro, Côte d'Ivoire \\ Kplohi Yaba Hervé, Msc \\ Féa Isaac, Msc \\ PETROCI, Centre d'Analyses et de Recherche (CAR), \\ Abidjan, Côte d'Ivoire
}

\begin{abstract}
In the present work, a mineralogical and chemical cartographic study of shelly limestones from the Lower Oligocene of Côte d'Ivoire Ivorian emerged basin was carried out. The analyzes were realized under a scanning microscope which is equipped with an energy diffusion spectrometer (SEMEDS). The objective is to understand the post-diagenetic processes which occurred in shelly limestones during Lower Oligocene Transgression. The results showed that shelly limestones are composed of more than $80 \%$ of calcium which are accompanied in order of abundance of silicon (6.73), sulfur (1.94\%), iron (1.62\%), aluminum (1.57\%) and magnesium $(1.44 \%)$. The results also reveal during the Lower Oligocene Transgression, mineralogical
\end{abstract}


inclusions were formed in chronological order: glauconite and pyrite. During this transgression, silicification (quartzification) of some bioclasts occurred between depths $3000 \mathrm{~m}$ and calcite compensation depth (CCD) explaining the presence of silica in these limestones by chemical micro-mapping. This new marine deposit (deeper sea) has caused the formation of hard-ground on shelly limestones surface.

Keywords: Silicification, Shelly Limestones, Chemical micro-mapping

\section{Introduction}

Les roches carbonatées sont des roches sédimentaires comportant au moins 50\% de carbonates. Elles peuvent être d'origine chimique (précipitation chimique) ou biogénique (accumulation de bioclastes). Parmi les roches carbonatées d'origine biogénique, on distingue les faluns et les lumachelles. Ces dernières sont issues principalement de l'accumulation sur place de bioclastes dans les environnements marins peu-profonds. Les calcaires lumachelliques sont connus dans le bassin sédimentaire émergé ivoirien plus précisément dans la localité d'Ebocco (DIGBEHI, 1987). Ils ont été mis en évidence dans des sondages des localités d'Eboïnda et d'Adiaké (ASSALE, 2013). Dans le sondage d'Adiaké, dont le profil lithologique est représenté en figure 1 et la localisation en figure 2, ces calcaires ont été identifiés entre les cotes $48 \mathrm{~m}$ et $52 \mathrm{~m}$ soit une épaisseur de $4 \mathrm{~m}$. Ces calcaires, datés de l'Oligocène inférieur (ASSALE, 2013), sont extrêmement durs de couleur gris jaunâtre à jaune foncé. La teneur en carbonate de calcium dans les calcaires lumachelliques d'Adiaké varie de 93,48 à 84,22\%. Ces calcaires sont encroutés sur une épaisseur d'un mètre (cotes 49 à $48 \mathrm{~m}$ ) formant ainsi un hard ground extrêmement dur qui traduit un arrêt momentané de la sédimentation du fait de la transgression. On pourrait se demander quels sont les phénomènes post-diagénétiques qui se sont produits dans ces calcaires au cours de cette transgression? C'est pour répondre à cette interrogation que le présent travail est initié. Son objectif principal est de comprendre les processus post-diagénétiques qui se sont produits dans les calcaires lumachelliques d'Adiaké au cours de la transgression de l'Oligocène inférieur. Les objectifs spécifiques assignés :

- faire une analyse chimique des calcaires lumachelliques d'Adiaké au spectromètre à diffusion d'énergie du MEB ;

- établir une cartographie chimique au MEB afin de comprendre la répartition des éléments chimiques dans ces calcaires. 
European Scientific Journal, ESJ

April 2021 edition Vol.17, No.14

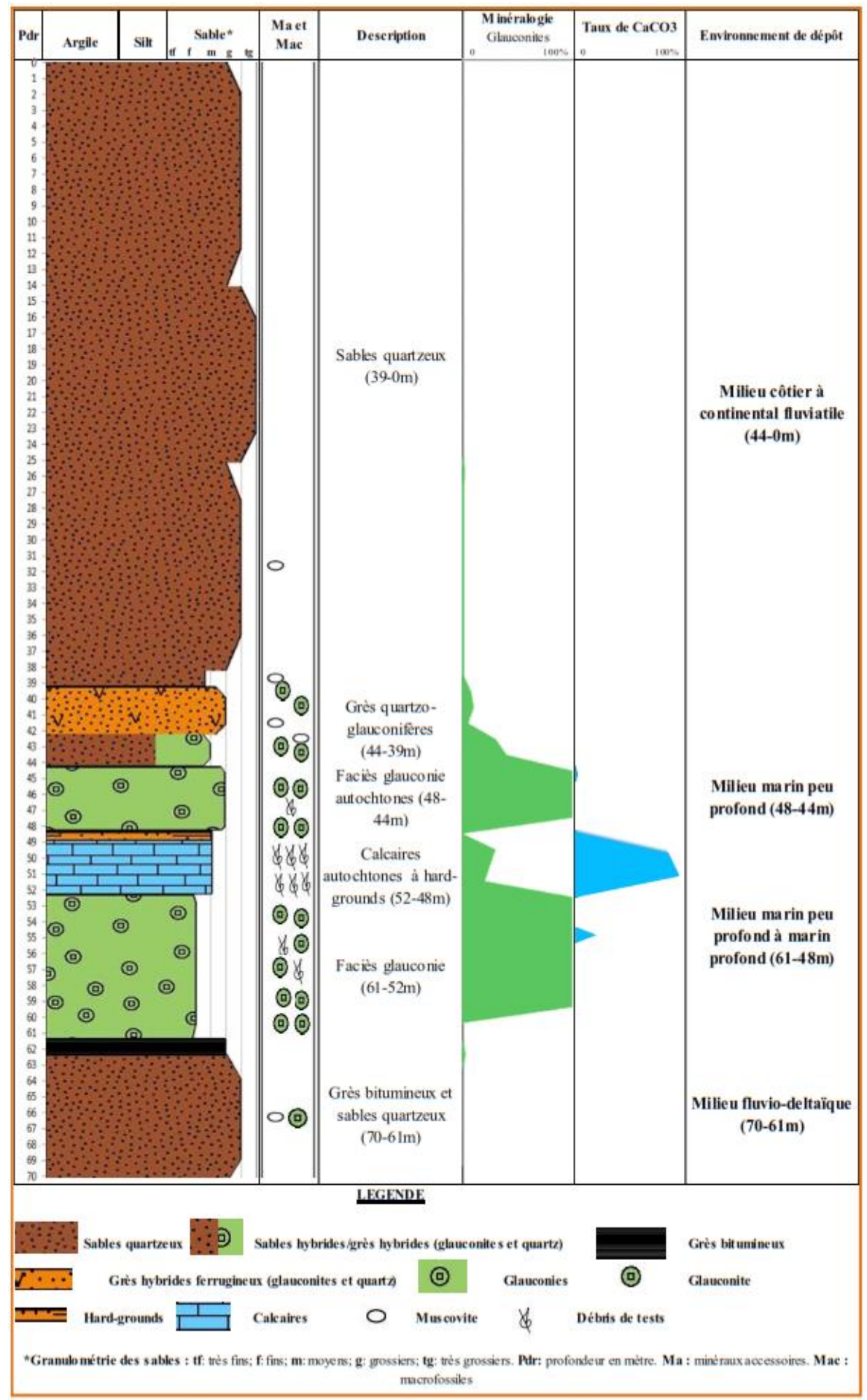

Figure 1 : Stratigraphie du puits Adia 5 (ASSALE, 2013) 


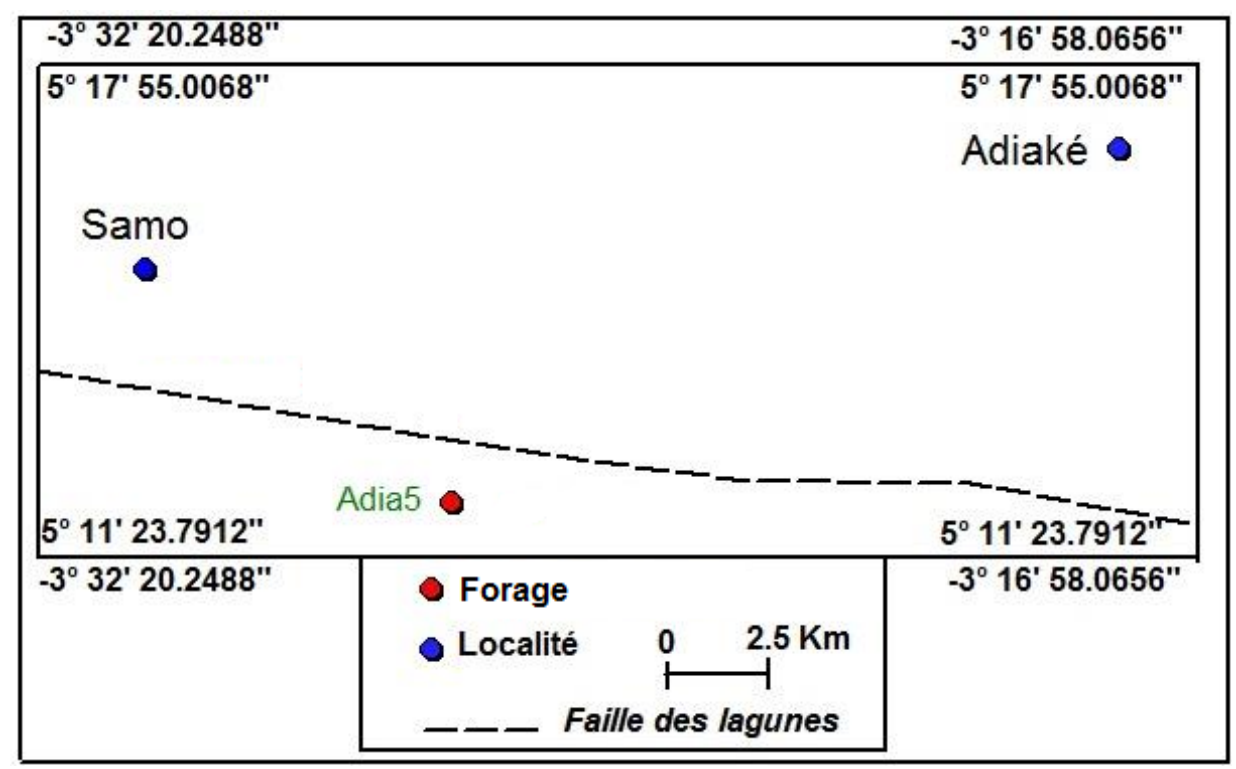

Figure 2 : Positionnement du forage Adia 5

\section{Matériel et Méthode}

Le matériel à analyser est constitué d'un morceau de carotte de roches carbonatées provenant du sondage Adia 5 du bassin onshore de Côte d'Ivoire. Cette carotte a été sélectionnée à la cote $50 \mathrm{~m}$ (Adia5-50). Avec cette carotte, une lame mince pétrographique non-couverte a été confectionnée (Photo 1). La lame est métallisée au carbone pour son analyse au MEB de type FEG Supra 40 VP de Zeiss équipé d'un spectromètre à diffusion d'énergie. Dans cette analyse, trois micro-surfaces d'environ $4 \mathrm{~mm}^{2}$ ont été sélectionnées. Pour chaque surface, une analyse chimique est effectuée en vue de la détermination des éléments chimiques majeurs présents. La précision de l'analyse chimique est de 10. Après cette analyse, une micro-cartographie est effectuée sur chaque surface. Elle consiste à attribuer à chaque élément chimique une couleur afin de comprendre leur répartition dans l'échantillon de roche. La proportion de carbone n'est pas prise en compte puisqu'il a été utilisé pour la métallisation de la surface de la lame.

Toutes ces analyses ont été effectuées au Centre d'Analyses et de Recherche de la PETROCI (Société des Opérations Pétrolières de Côte d'Ivoire). 


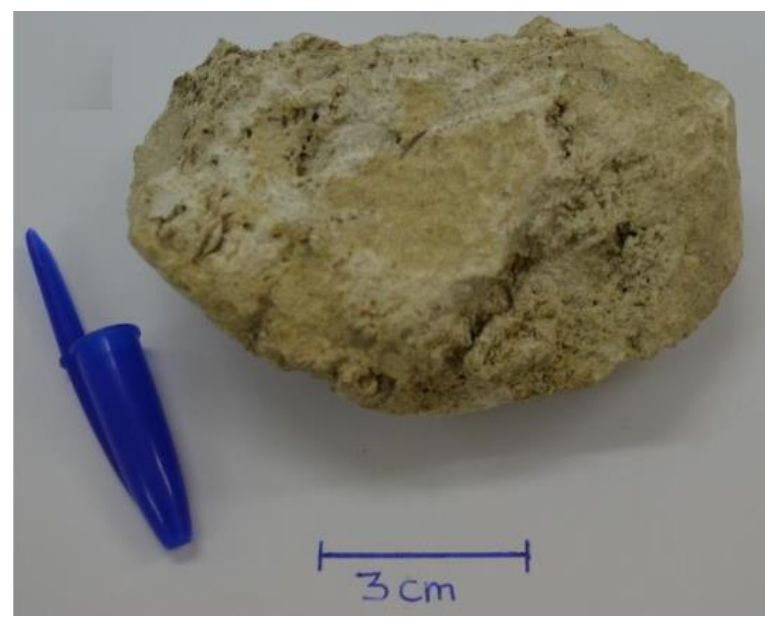

Photo 1 : Calcaire lumachellique de la cote $50 \mathrm{~m}$ (ASSALE, 2013)

\section{Résultats}

\subsection{Composition géochimique du calcaire Adia5-50} le tableau 1.

La composition chimique des trois micro-surfaces est consignée dans

Tableau 1 : Composition chimique des micro-surfaces du calcaire Adia5-50

\begin{tabular}{|l|c|c|c|c|c|c|c|}
\hline Micro-surfaces & $\mathbf{M g}$ & $\mathbf{A l}$ & $\mathbf{S i}$ & $\mathbf{S}$ & $\mathbf{C a}$ & $\mathbf{F e}$ & Total \\
\hline Micro-surface 1 & 1,42 & 1,64 & 6,24 & 1,82 & 87,52 & 1,36 & 100 \\
\hline Micro-surface 2 & 1,44 & 1,22 & 6,41 & 1,42 & 88,19 & 1,32 & 100 \\
\hline Micro-surface 3 & 1,45 & 1,85 & 7,55 & 2,59 & 84,37 & 2,19 & 100 \\
\hline Moyenne & $\mathbf{1 , 4 4}$ & $\mathbf{1 , 5 7}$ & $\mathbf{6 , 7 3}$ & $\mathbf{1 , 9 4}$ & $\mathbf{8 6 , 6 9}$ & $\mathbf{1 , 6 2}$ & \\
\hline
\end{tabular}

L'analyse de ces micro-surfaces au MEB-EDS montre que le calcaire Adia5-50 est constitué de six éléments chimiques majeurs à savoir le magnésium, l'aluminium, le silicium, le soufre, le calcium et le fer. Dans cette composition chimique, le calcium est l'élément prépondérant avec une moyenne de $86,69 \%$ (Figure 3 ). Il est suivi par le silicium dont la proportion oscille entre $6,24 \%$ et $7,55 \%$ avec une moyenne de $6,73 \%$. Les autres éléments chimiques ont des proportions n'excédant pas $2 \%$. Au vue de l'analyse chimique, cette roche est bel et bien un calcaire.

\subsection{Micro-cartographie chimique des éléments principaux du calcaire Adia5-50 \\ Dans la micro-cartographie, les couleurs attribuées aux éléments chimiques sont présentées dans le tableau 2. En plus des six éléments chimiques, le carbone et l'oxygène ont été pris en compte dans cette cartographie ; ce qui donne un total de huit éléments chimiques.}




\section{Moyenne}

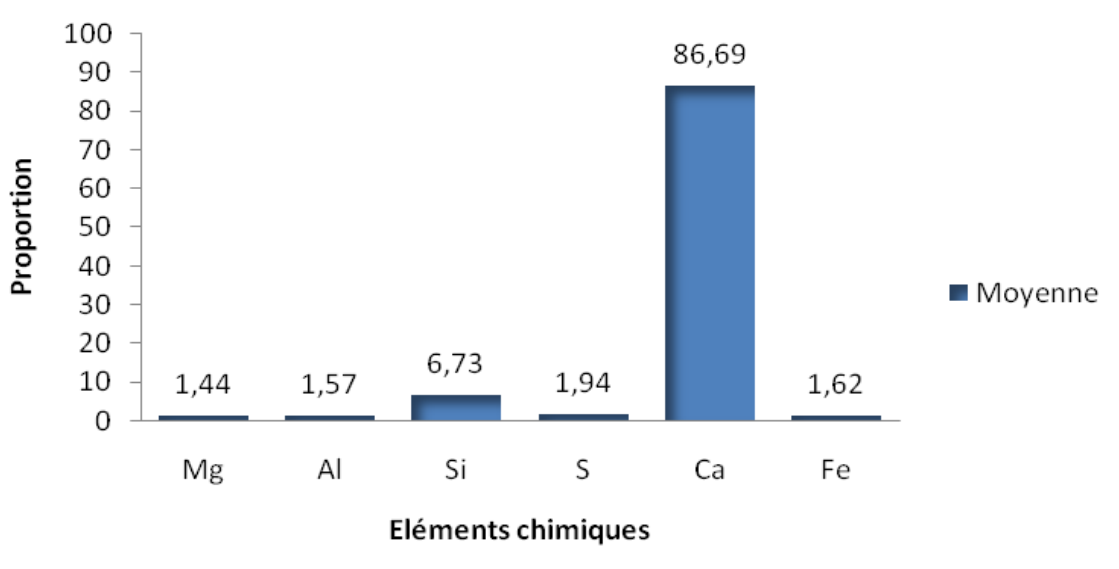

Figure 3 : Proportion des elements chimiques presents dans le calcaire Adias-50m.

Tableau 2 : Couleurs attribuées aux éléments chimiques du calcaire

\begin{tabular}{|c|c|c|c|c|c|c|c|c|}
\hline Eléments & $\mathbf{C}$ & $\mathbf{O}$ & $\mathbf{M g}$ & $\mathbf{A l}$ & $\mathbf{S i}$ & $\mathbf{S}$ & $\mathbf{C a}$ & $\mathbf{F e}$ \\
\hline Couleur & $\begin{array}{c}\text { gris } \\
\text { argenté }\end{array}$ & $\begin{array}{c}\text { blanc } \\
\text { argenté }\end{array}$ & $\begin{array}{c}\text { violet } \\
\text { clair }\end{array}$ & bleu foncé & rose & jaune & verte & rouge \\
\hline $\begin{array}{c}\text { Numéro photo } \\
\text { sur la planche }\end{array}$ & Photo 2 & Photo 3 & Photo 4 & Photo 5 & Photo 6 & Photo 7 & Photo 8 & Photo 9 \\
\hline
\end{tabular}

Pour chaque micro-surface, une planche constituée de 9 photos a été réalisée. Sur chaque planche, la première photo (haut à l'extrême gauche) constitue la surface sélectionnée comportant les éléments chimiques. Les huit autres photos représentent l'image de la dispersion des huit éléments chimiques. Ces huit photos suivent l'ordre présenté dans le tableau 2.

La micro-surface 1 montre que les éléments chimiques tels que le carbone, l'oxygène, le magnésium, l'aluminium, le silicium, le soufre, le calcium et le fer sont répartis uniformément dans le calcaire (Planche 1). Parmi ces éléments, le carbone, le soufre et le fer présentent des concentrations anormales dans le calcaire. La présence et la concentration anormale du carbone (cercle rouge de la photo 2) est due à la métallisation. Les concentrations anormales de soufre et de fer (cercles bleus des photos 7 et 9) révèlent la présence de pyrite dans le calcaire Adia5-50. Quant au silicium, il se retrouve concentré à certains endroits sur le calcaire témoignant ainsi de la silicification du calcaire (photo 6). Sur la photo 8 (cercle rouge), représentant la dispersion du calcium, on remarque des espaces noirs qui correspondent au carbone concentré anormalement (cercles rouges) et à la silice (espaces noirs non entourés). 


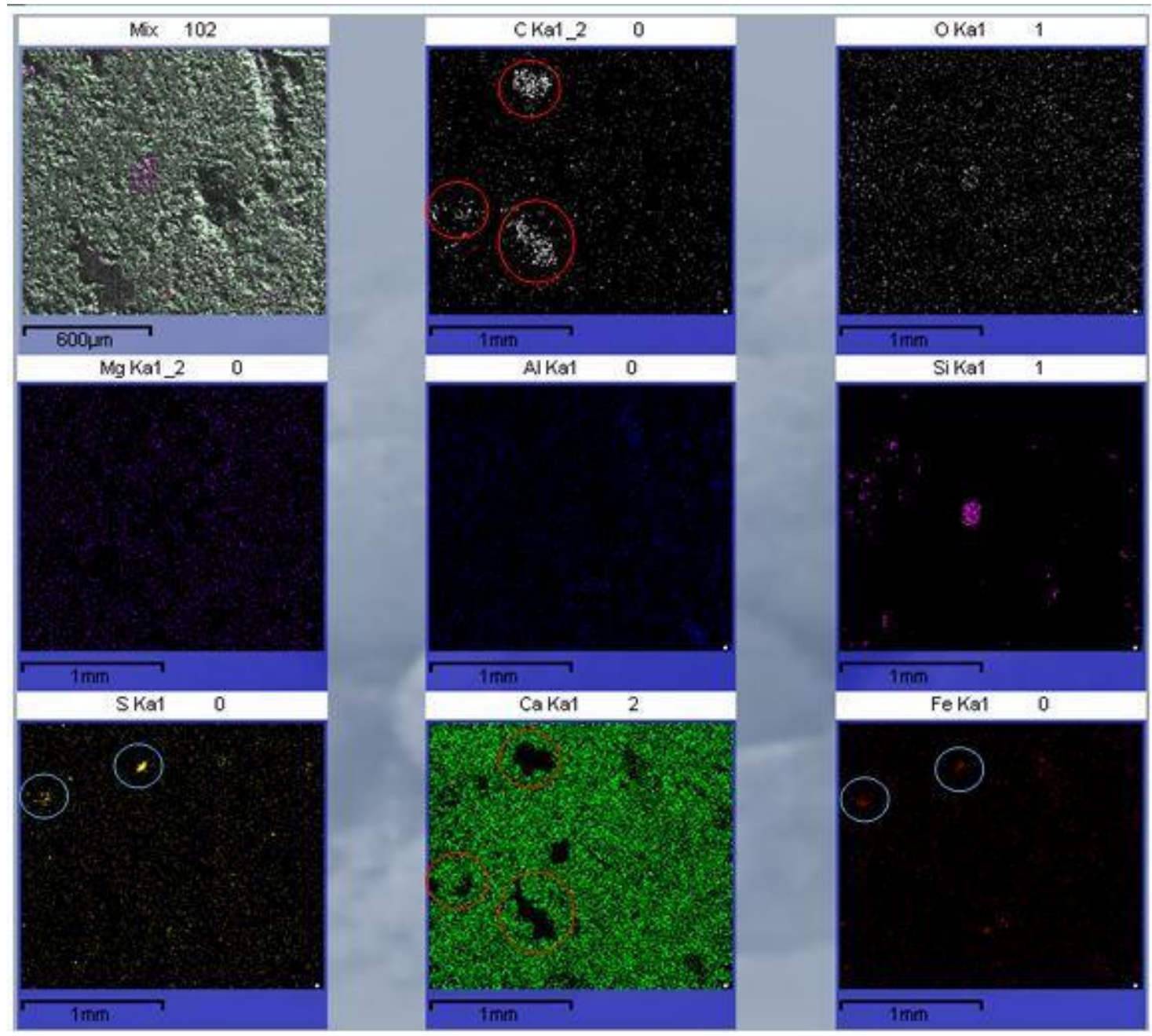

Planche 1 : Cartographie chimique de la zone 1 du calcaire Adia5-50m

Sur la micro-surface 2 (Planche 2), seuls le silicium, l'aluminium et le fer sont concentrés par endroits. Les photos 7 et 9 (cercle jaune) témoignent de la présence de la pyrite. Les autres zones de concentrations de fer et d'aluminium sont dues à l'oxydation du calcaire qui a favorisé la concentration de ces deux éléments chimiques ; cela a été possible grâce aux substitutions de ces éléments avec le calcium. La concentration du silicium suggère la silicification du calcaire suite aux substitutions entre le silicium et le calcium. Les zones noires de la photo 8 (photo du calcium) correspondent aux ombres visibles sur la photo 3 . 


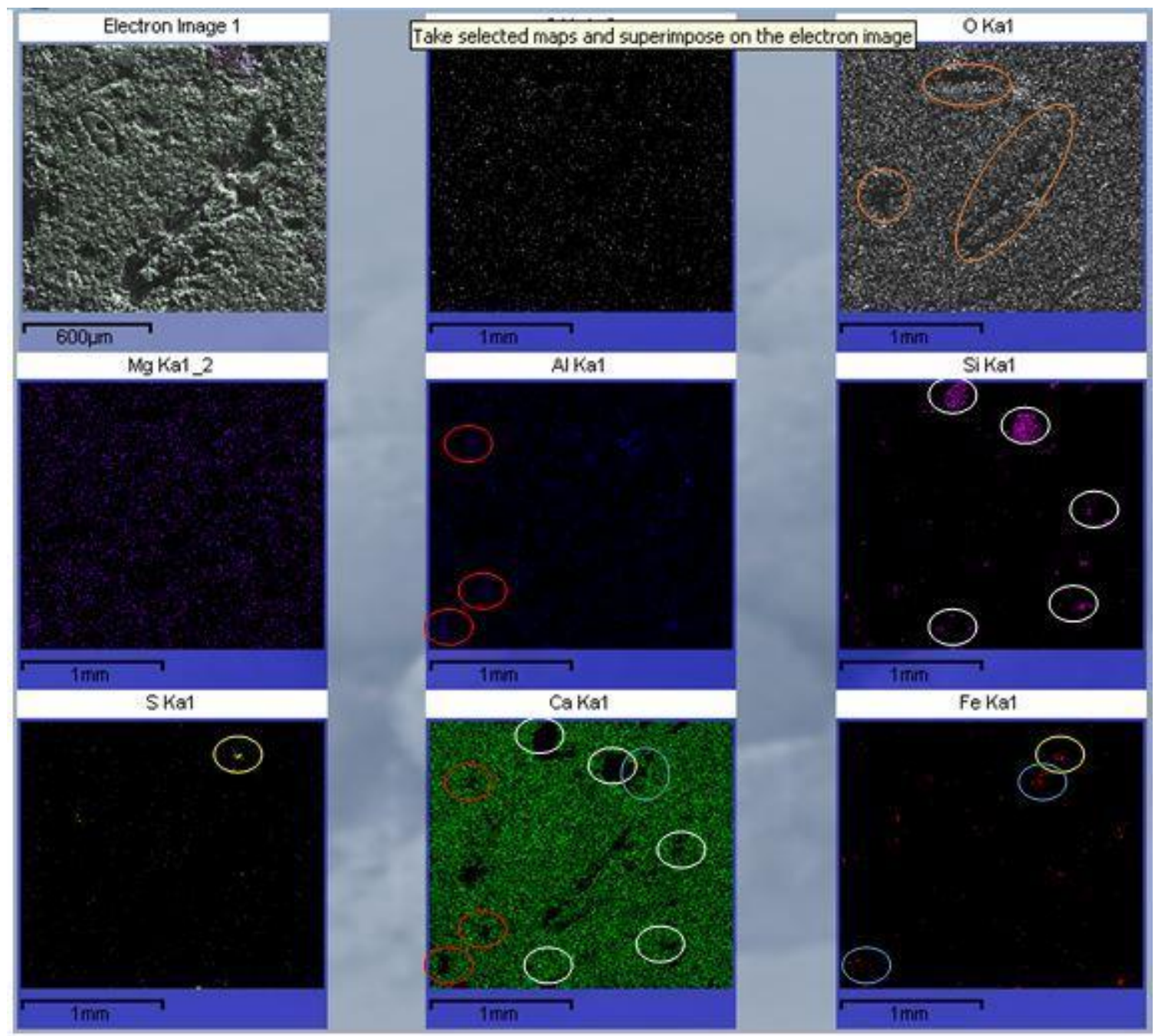

Planche 2 : Cartographie chimique de la zone 2 du calcaire Adia5-50m

La micro-surface 3 est comparable à la micro-surface 2 avec également des concentrations de silicium, de fer, d'aluminium et de soufre (Planche 3). La concentration du soufre est due à la présence de pyrite. Sur cette microsurface, le silicium est plus concentré que sur les deux autres micro-surfaces. La silicification est donc plus prononcée sur cette surface. 


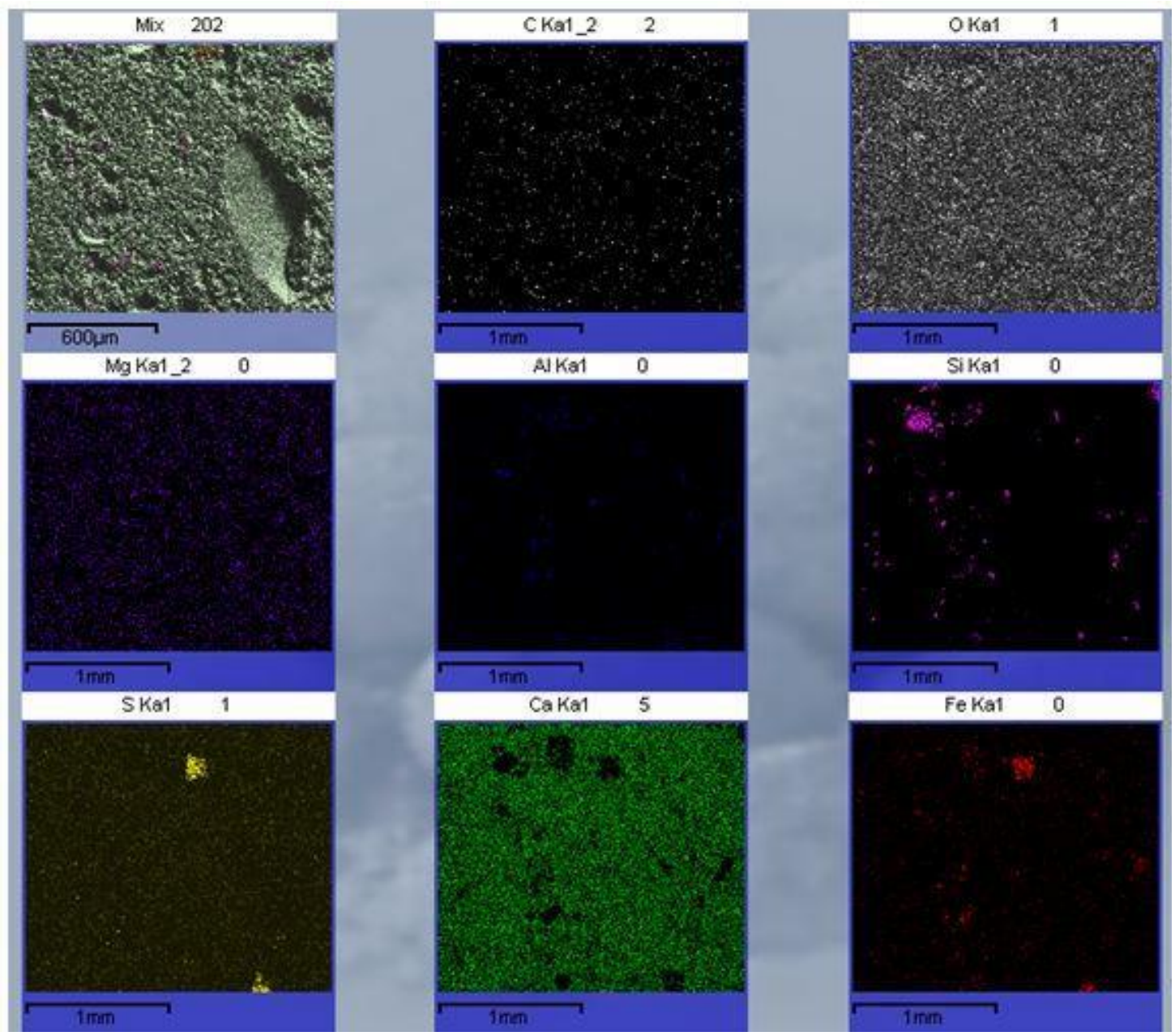

Planche 3 : Cartographie chimique de la zone 3 du calcaire Adia5-50m

\section{Discussion}

Les calcaires lumachelliques étudiés sont constitués de l'accumulation de bioclastes in situ (lumachelles). Cela indique l'existence d'un récif développé sur un plateau continental marginal (MAURICE, 2003). La taphonomie des organismes résulte de la transgression créée au cours de l'Oligocène inférieur occasionnant ainsi l'approfondissement d'eaux marines et la réduction de la lumière (ASSALE 2013). Cet auteur confirme que la transgression a transformé l'environnement marin peu profond à un milieu marin plus profond. La silicification observée dans cette étude a donc été favorisée par cette transgression. L'environnement marin profond se situe entre $3000 \mathrm{~m}$ et la zone de compensation des carbonates (CCD). Selon GARY (2009), la dissolution des calcaires biogéniques débutent à partir de $3000 \mathrm{~m}$. Cette dissolution est complète dans les profondeurs avoisinant les $4000 \mathrm{~m}$ à 
savoir dans la zone de compensation de la calcite (WISE, 2003). A ces profondeurs, le $\mathrm{pH}$ est basique et la température basse (GARY, 2009). Ces conditions favorisent la précipitation de la silice (THIRY et al., 1988, GERARD, 2003) : d'où des conditions favorables pour une silicification des calcaires biogéniques (MAURICE, 2003). La silicification mise en évidence de cette étude conforte donc la manifestation d'une transgression au cours de l'Oligocène inférieure dans le bassin ivoirien signalé dans les travaux de ASSALE (2013). Dans les travaux pétrographiques réalisés sur ces calcaires par cet auteur, des coquilles entière de macrofossiles se sont transformées en quartz; la silicification est donc ici une quartzification. La pyrite s'est également formée dans les calcaires durant cette période bien après la formation des glauconites du moment où une abondance de soufre dans le milieu sédimentaire empêche la glauconitisation (ASSALE et al., 2020). La production du soufre a favorisé le soufrage des calcaires ; ce qui explique la présence du soufre dans la composition chimique de ces calcaires. Les autres concentrations à savoir le fer et l'aluminium et peut-être le magnésium se sont produites durant l'encroûtement de ces calcaires puisque leur surface est constituée d'hard-ground. La quartzification traduit donc une diagenèse par recristallisation.

\section{Conclusion}

Les analyses effectuées dans le présent travail montrent que les calcaires lumachelliques de l'Oligocène inférieur du bassin sédimentaire ivoirien proviennent de l'accumulation in situ de bioclastes. L'étude minéralogique montre que ces carbonates sont composés principalement de calcium $(86,69 \%)$ qui sont accompagnés par ordre d'abondance de silicium $(6,73)$, de soufre $(1,94 \%)$, de fer $(1,62 \%)$, d'aluminium $(1,57 \%)$ et de magnésium (1,44\%). Au niveau de la micro-cartographie chimique, on remarque que les éléments chimiques tels que le magnésium, l'aluminium et le fer sont répartis uniformément dans ces calcaires. Néanmoins, des concentrations de fer, de soufre, de magnésium, d'aluminium et de silicium s'y rencontrent. Les concentrations du soufre se superposent aux concentrations du fer; ce qui témoigne de la présente de pyrite dans les calcaires étudiés. Les concentrations de fer non associées au soufre et celles de l'Alluminium et du Magnésium sont du fait de encroûtement de la surface des calcaires lors de la transgression Oligocène. C'est durant cette transgression que la silicification (quartzification) des bioclastes s'est produite entre les profondeurs $3000 \mathrm{~m}$ et la CDD.

\section{References:}

1. Assalé F. Y. P. (2013). Caractérisation sédimentologique, palynologique, géochimique et paléoenvironnementale des formations 
connexes à la faille des lagunes (Est du bassin onshore de Côte d'Ivoire). Thèse Doctorat uniq. Univ. F.H.B, Côte d'Ivoire, 361p.

2. Assalé F. Y. P., Akobe A. C., Amani E. M. et Kplohi Y. H. (2020). Évolution de la glauconitisation dans le bassin offshore ivoirien : étude chimique et ultrastructurale des glauconites. Géologie de la France, $\mathrm{n}^{\circ}$ 2, p. 26-40.

3. Digbéhi Z. B. (1987). Études comparées de la sédimentation des premiers stades d'ouvertures de l'Atlantique - Golfe de Guinée Golfe de Gascogne. Sédimentologie, Biostratigraphie. Thèse de doctorat, Univ. Pau, 366p.

4. Gary N. S. (2009). Sedimentology and stratigraphy. Second edition. Wiley-Blackwell : A John Wiley \& Sons, Ltd., Publication 111 River Street, Hoboken, USA, 419p.

5. Gerard V. M. (2003). Encyclopedia of Earth Sciences Series : Encyclopedia of sediments and sedimentary rocks. Edition Springer, Canada, 821p.

6. Maurice E. T., 2003. Sedimentary Rocks in the Field. Third EDITION. Department of Geological Sciences University of Durham, UK. John Wiley \& Sons Ltd, England, 234p.

7. Thiry M., Koeniguer J-C., Menillet F. (1988). Les silicifications de surface : la typologie et les outils de leur interprétation. Bull. Inf. Géol. Bass. Paris, Vol. 25, N4, p. 5-14.

8. Wise, S. (2003). Calcite compensation depth. In: Encyclopedia of Sediments and Sedimentary Rocks (Ed. Middleton, G.V.). Kluwer Academic Publishers, Dordrecht; 88-89. 